\title{
Fish, marine $n-3$ fatty acids, and atrial fibrillation - experimental data and clinical effects
}

\author{
Thomas Andersen Rix ${ }^{1}{ }^{*}$, Lotte Maxild Mortensen ${ }^{\mathbf{1}}$ and Erik Berg Schmidt ${ }^{1}$ \\ ${ }^{1}$ Department of Cardiology, Aalborg AF Study Group, Center for Cardiovascular Research, Aalborg Hospital, Aarhus University Hospital, Aalborg, Denmark \\ 2 Section of Epidemiology, Department of Public Health, Aarhus University, Aarhus, Denmark
}

Edited by:

George E. Billman, The Ohio State

University, USA

Reviewed by:

David R. Van Wagoner, Cleveland Clinic Lerner College of Medicine of

Case Western Reserve University,

USA

Cynthia Carnes, The Ohio State

University, USA

${ }^{*}$ Correspondence:

Thomas Andersen Rix, Department of

Cardiology, Aalborg Hospital, Sdr.

Skovvej 15, 9000 Aalborg, Denmark.

e-mail: tar@rn.dk
Marine $n-3$ polyunsaturated fatty acids (PUFA) may have beneficial effects in relation to atrial fibrillation (AF) with promising data from experimental animal studies, however, results from studies in humans have been inconsistent. This review evaluates the mechanisms of action of marine $n-3$ PUFA in relation to AF based on experimental data and provides a status on the evidence obtained from observational studies and interventional trials. In conclusion, there is growing evidence for an effect of marine n-3 PUFA in prevention and treatment of AF. However, further studies are needed to establish which patients are more likely to benefit from n-3 PUFA, the timing of treatment, and dosages.

Keywords: atrial fibrillation, fish, marine $n-3$ polyunsaturated fatty acids

\section{INTRODUCTION}

Faced with the growing epidemic of atrial fibrillation (AF), there is an unmet need for preventive measures as well as better treatment of this disorder. Some of the advances in recent years have been on rhythm control to restore sinus rhythm, but the development of effective and safe drugs for restoring sinus rhythm has been difficult, trying to balance beneficial anti-arrhythmic effects with risk of pro-arrhythmic adverse effects. Invasive intervention by catheter ablation techniques and surgery may be more effective, but is an option mostly for highly symptomatic patients, given the risk of peri-procedure complications and high costs. Fish consumption and intake of marine $n-3$ polyunsaturated fatty acids (PUFA), with suggested anti-arrhythmic effects in relation to malignant ventricular arrhythmias, is safe and with few side-effects.

This review will discuss the mechanisms of action of marine $n-3$ PUFA in relation to AF based on experimental data and provide a status on the evidence of the effect of marine $n-3$ PUFA in $\mathrm{AF}$ as obtained from observational studies and interventional trials.

\section{BACKGROUND}

\section{FISH, MARINE $n-3$ PUFA, AND CARDIAC DISEASE}

In early studies of Greenland natives, Bang, and Dyerberg found a lower risk of death from coronary heart disease (Dyerberg et al., 1978). The Greenland Eskimos were living on a diet consisting largely on whales, seals, and fish, thus consuming around $10-14 \mathrm{~g} /$ day of marine $n-3$ PUFA, whereas most western populations consume less than $0.5 \mathrm{~g} /$ day (De Caterina, 2011). Several epidemiological studies have confirmed the finding in Greenland Eskimos, although data are not entirely consistent (Bjerregaard et al., 2010; De Caterina, 2011; Mozaffarian and Wu, 2011). Marine $n-3$ PUFA may affect the risk of cardiovascular disease by a long list of mechanisms including a lowering of triglycerides, a reduction in blood pressure, together with antithrombotic and anti-inflammatory effects (De Caterina, 2011; Mozaffarian and $\mathrm{Wu}, 2011)$. Furthermore, in some studies marine $n-3$ PUFA have lowered the risk of sudden cardiac death (De Caterina, 2011; Mozaffarian and Wu, 2011) and in turn, these observed effects on ventricular tachyarrhythmia have lead to research into potential effects of $n-3$ PUFA on atrial rhythm disturbances including AF.

\section{MARINE $n-3$ PUFA}

Polyunsaturated fatty acids are divided into $n-3$ (omega-3) and $n-6$ according to the position of the first double bond. Both groups are essential fatty acids, and therefore the content in the human body is (almost) fully dependent on dietary intake. The $n-3$ PUFA family consists of alpha-linolenic acid (ALA) derived from plants and the marine $n-3$ PUFA consisting of eicosapentaenoic acid (EPA), docosahexaenoic acid (DHA), and docosapentaenoic acid (DPA). Marine $n-3$ PUFA are most abundant in fatty fish and in the liver of lean fish (Calder, 2012). In addition to their dietary intake, EPA, DHA, and DPA can be synthesized endogenously from ALA but only to a very limited extent (Burdge and Calder, 2005). The biologically most important $n-3$ PUFA are thought to be EPA and DHA, while very little is known about the effect of DPA.

\section{ATRIAL REMODELING}

Atrial remodeling is defined as any persistent change in atrial structure or function (Nattel et al., 2008) that may occur either as a substrate induced by another underlying condition or secondary to AF itself. For AF to develop, some fundamental arrhythmia mechanisms are required; both a suitable substrate and some form of triggering activity, and these, in turn, facilitate and initiate reentry of electrical impulses, thereby maintaining AF (Nattel et al., 
2008). Atrial remodeling can increase the potential for ectopic and reentrant activity by a variety of mechanisms (Nattel et al., 2008).

Ectopic firing provides a trigger for reentry, but may also in itself maintain AF in the case of a rapid ectopic firing focus (Nattel et al., 2008). Abnormalities in cellular $\mathrm{Ca}^{2+}$ handling can cause ectopic firing, e.g., because of delayed after-depolarizations related to $\mathrm{Ca}^{2+}$ overload.

Ischemia, inflammation, and dilation make atria more vulnerable to AF (Nattel et al., 2008). For reentry to be maintained, the cells must have regained their excitability when the impulse has traversed the circuit, and therefore reentry is favored by shortening of the refractory period (e.g., by rapid atrial activation in $\mathrm{AF}$ ), slow conduction of the impulse (e.g., by changes in cellular and tissue structure including fibrosis), and longer distance of the impulse circuit (e.g., atrial enlargement). The fibrillatory activity may occur either because of an irregular atrial response to a single reentry circuit or because of multiple simultaneous functional reentry circuits (Nattel et al., 2008).

Atrial remodeling can be divided into two major groups according to whether it is related to a rapid atrial rate such as in AF, or caused by an underlying condition. Rapid atrial tachycardia results in shortening of the atrial refractory period (electrical remodeling), occurs within the first few days of AF, and is reversed within 1 week after reversion to sinus rhythm (Allessie et al., 2002). Secondly, AF also results in impaired contractility (contractile remodeling) of the atria after reversion to sinus rhythm and depending on the duration of AF, and recovery is slower lasting days to months (Allessie et al., 2002). Atrial structural remodeling refers to structural changes in the atria and may occur either during prolonged atrial tachycardia or caused by different cardiac pathologies such as hypertensive heart disease, heart failure, myocardial infarction, and cardiomyopathy. It includes histological changes in the atrial myocytes in terms of dedifferentiation, and the associated atrial fibrosis causes intra-atrial conduction disturbances (Allessie et al., 2002). AF increases dramatically with older age, which is likely in part mediated by age-related fibrosis, changes in conduction, and increased likelihood of block (Dun and Boyden, 2009).

\section{METHODS}

\section{SEARCH FOR LITERATURE}

We searched PubMed using the MesH terms "AF" and ("Seafood," "Fatty Acids, Omega-3," "Fish Oils," "Fishes," or "Dietary Fats") and EMBASE using the terms "heart atrium fibrillation" and ("omega3 fatty acid," "sea food," "fish oil," or "exp fish"). Reference lists from identified reports were checked for additional publications. Criteria for consideration were publications in English published until February 2012. All identified abstracts were assessed, and relevant published reports were identified. We discarded results only published as abstracts.

\section{MARINE $n-3$ PUFA AND CARDIAC ARRHYTHMIAS MECHANISMS OF ACTION}

The effects of marine $n-3$ PUFA in relation to ventricular arrhythmia have been extensively studied whereas few have studied the effects on atrial myocytes. For this reason, some mechanisms of action related to ventricular arrhythmia are summarized in order to further suggest possible effects of $n-3$ PUFA on atrial myocytes.
As recently reviewed (Saravanan et al., 2010b; Mozaffarian and Wu, 2011), $n-3$ PUFA have beneficial effects on various cardiovascular risk factors, inflammation, and effects related to arrhythmia via several mechanisms including effects on cell and organelle membrane structure and function; ion channels and electrophysiology; and nuclear receptors and transcription factors.

Anti-arrhythmic effects of a diet rich in marine $n-3$ PUFA were studied in a rat model by McLennan (1993) where these fatty acids were shown to prevent ventricular fibrillation in rats, and, in similar experiments in marmoset monkeys, they increased the thresholds for ventricular fibrillation (McLennan et al., 1993).

Likewise, subsequent studies of intravenous infusion of $n-3$ PUFA in a dog model of ventricular arrhythmia showed that ventricular fibrillation was much more difficult to elicit after infusion of marine $n-3$ PUFA (Billman et al., 1999). In a series of in vitro studies, Leaf et al. (Leaf et al., 2005; Xiao et al., 2005) demonstrated anti-arrhythmic effects of marine $n-3$ PUFA. Thus, $n-3$ PUFA affect numerous ion channels such as inhibitory effects on the inward sodium current $\left(I_{\mathrm{Na}}\right)$ and $\mathrm{L}$ type inward calcium current $\left(I_{\mathrm{Ca}, \mathrm{L}}\right)$ which may be important for their anti-arrhythmic effects (Leaf et al., 2005). n-3 PUFA affect the $I_{\mathrm{Na}}$ by shifting the steady-state inactivation to hyperpolarized potentials and thereby prolonging the refractory period (Xiao et al., 1998, 2000). Inhibition of $I_{\mathrm{Ca}, \mathrm{L}}$ by $n-3$ PUFA (Xiao et al., 1997) prevents triggering activity from after-potential discharges caused by excessive cytosolic $\mathrm{Ca}^{2+}$ fluctuations (Leaf et al., 2005). Also, $n-3$ PUFA have electrical stabilizing effects on myocytes as shown in vitro (Kang and Leaf, 1996) where myocytes added EPA showed reduced spontaneous beating rate and required higher electrical stimulation to contract (Leaf et al., 2005).

\section{ACUTE VS. CHRONIC EFFECTS}

n-3 PUFA may principally operate in at least two different ways, either circulating in the blood or after incorporation into cells. Studies on circulating $n-3$ PUFA typically have used experimental designs where $n-3$ PUFA is added intravenously or in vitro in cellular studies and may thus be termed "acute effects," whereas "chronic effects" reflect dietary exposure over time where $n-3$ PUFA is incorporated into cellular membranes in addition to a steady-state of circulating $n-3$ PUFA (comparatively less than when administered intravenously). The period to full incorporation of $n-3$ PUFA has been reported to be 28 days for myocardium content of DHA in rats (Owen et al., 2004), 12 weeks for incorporation of EPA and DHA in right atrium and left ventricle in dogs (Billman et al., 2010), and 30 days for DHA and EPA in right atrial appendage tissue in humans (Metcalf et al., 2007). Importantly, "acute effects" of circulating $n-3$ PUFA have been shown to be different from "chronic effects" of $n-3$ PUFA in cellular membranes, which include different effects on various ion channels as reviewed by Den Ruijter and Coronel (2009). For example, circulating $n-3$ PUFA inhibit $I_{\mathrm{Na}}$ and $I_{\mathrm{Ca}, \mathrm{L}}$, whereas $n-3$ PUFA incorporated into cellular membranes do not affect $I_{\mathrm{Na}}$, but both inhibit $I_{\mathrm{Ca}, \mathrm{L}}$ and the reopening of the calcium channel at plateau potentials. Thus, when interpreting clinical studies on $n-3$ PUFA, it is important to distinguish between studies on acute intravenous and long-term dietary exposure. 


\section{FISH, MARINE $n-3$ PUFA, AND ATRIAL FIBRILLATION POTENTIAL MECHANISMS}

Among these general effects on cardiac arrhythmia, some mechanisms may be of particular interest in relation to AF. Some mechanisms underlying AF include triggering activity by rapidly firing focal ectopic sources (often from the pulmonary veins) and reentry mechanisms including functional reentry circuits (probably more predominant in early stages such as paroxysmal AF) and multiple-circuit reentry (probably related to more pronounced structural remodeling in patients with persistent AF). $n-3$ PUFA reduce triggering activity and may affect reentry mechanisms in ventricular myocytes (Den Ruijter et al., 2007), and similar effects may occur in atrial myocytes (Nattel and Van Wagoner, 2011). This is, however, yet to be established.

Also, marine $n-3$ PUFA affect risk factors for ischemic heart disease such as lowering of blood pressure and plasma triglycerides and have anti-inflammatory effects, as they compete directly with $n-6$ PUFA as substrates for inflammatory eicosanoids, with the $n$-3-derived leukotrienes and thromboxanes being less inflammatory than products derived from $n-6$ PUFA (Calder, 2006).

Thus, the effects of marine $n-3$ PUFA on AF may be via mechanisms related to atherosclerosis and ischemic heart disease, including anti-inflammatory effects as well as direct anti-arrhythmic effects on myocytes through effects on ion channels, electrical stabilizing effects, and fluidity of the cell membrane.

\section{EXPERIMENTAL ANIMAL STUDIES}

In an in vitro model, induction of asynchronous contractile activity by a $\beta$-adrenoceptor stimulus in rat atrial myocytes was reduced by addition of DHA and EPA, while cell membrane fluidity was increased (Jahangiri et al., 2000). Also, EPA reduced arrhythmogenesis in isolated rabbit pulmonary vein tissue via nitric oxide production (Suenari et al., 2011).

Electrical remodeling by reduction of atrial refractory period is an important early remodeling event that favors the development and maintenance of AF. In a model of stretch-induced vulnerability of AF, rabbits fed $n-3$ PUFA were less susceptible to induce and sustain AF and also had less stretch-induced shortening of atrial refractory period (Ninio et al., 2005). Infusion of $n-3$ PUFA significantly reduced the shortening of atrial refractory period in an experimental model on dogs receiving rapid atrial pacing (da Cunha et al., 2007), whereas this was not found in a different study on dogs fed $n-3$ PUFA for a longer duration (Sakabe et al., 2007). Effects on structural remodeling were investigated in a ventricular tachypacing model of congestive heart failure. Dogs fed $n-3$ PUFA developed less atrial structural remodeling in terms of atrial fibrosis and conduction abnormalities as well as shorter duration of burst pacing-induced AF (Sakabe et al., 2007). Similar results were found in rabbits fed a diet of purified EPA which showed suppression of atrial structural remodeling with less cardiac fibrosis, shorter duration of induced AF, as well as a less inflammatory profile in atrial and epicardial adipose tissues (Kitamura et al., 2011). Also, in a model with 2 weeks of simultaneous atrioventricular pacing, dogs fed $n-3$ PUFA had less AF inducibility and shorter episodes of AF, reduced conduction anisotropy in the left atrium, and prevention of pacing-induced increase in collagen turnover and collagen deposition in atrial appendage (Laurent et al., 2008). Furthermore, there was a beneficial effect on genes related to fibrosis, hypertrophy, and inflammation (Ramadeen et al., 2010).

In a model of post-operative AF in dogs fed $n-3$ PUFA for 3 weeks before excision of the left atrial appendage, AF was not inducible (0/7) compared to induction in four of six control animals. In addition, $n-3$ PUFA-treated animals had longer postoperative atrial effective refractory period, increased heart rate variability, and reduced atrial inflammation (Mayyas et al., 2011). Likewise, in a model of AF induced by sterile pericarditis, dogs fed $n-3$ PUFA for 4 weeks had less inflammation and reduced inducibility and maintenance of AF (Zhang et al., 2011). Finally, in a model of vagally induced AF, dogs fed $n-3$ PUFA were less vulnerable to develop AF (Sarrazin et al., 2007).

Given the multiple disposing factors for developing AF with older age in humans, the type of experimentally induced AF in different animal models is a major limitation for interpreting the results in addition to the inherent differences between species. Also, acute effects of $n-3$ PUFA are tested by in vitro or intravenous administration of $n-3$ PUFA, whereas chronic effects are investigated in the models where animals have been fed $n-3$ PUFA. However, while the reported effects of treatment in these models suggest that they may serve as a useful tool for investigating possible effects of $n-3$ PUFA in relation to AF, human studies are needed to establish whether marine $n-3$ PUFA are clinically useful in this population.

Taken together, data from animal studies on AF show convincing results with a substantial effect of $n-3$ PUFA, and for some parameters there is almost a complete reduction of the differences induced in the experimental models. Thus, the effects of $n-3$ PUFA in these studies include reduction in the shortening of atrial refractory period (electrical remodeling), reduction of atrial fibrosis (structural remodeling), reduction of post-operative AF (inflammation), and a reduction in vagally induced AF.

\section{PRIMARY PREVENTION OF AF}

A number of observational studies have addressed whether intake of fish and marine $n-3$ PUFA was associated with a lower risk of developing AF (Table 1). Promising data on fish consumption and prevention of AF were originally found in a prospective cohort from the Cardiovascular Health Study (Mozaffarian et al., 2004). The 4815 study participants were 65 years or older, and during 12 years of follow-up, 980 incident cases of AF occurred. Consumption of tuna or other broiled or baked fish was associated with a $28 \%$ lower risk of AF with intake one to four times per week (HR 0.72, 95\% CI 0.58-0.91, $p=0.005$ ), and 31\% lower risk with intake $\geq 5$ times per week (HR 0.69, 95\% CI 0.52-0.91, $p=0.008$ ), compared to intake less than once per month. In contrast, consumption of fried fish or fish sandwiches was not correlated to plasma $n-3$ PUFA levels and was suggestive of a higher risk of AF although not statistically significant. These types of fish primarily include lean fish and in addition, the method of cooking influences the fatty acid content as frying has been reported to affect the fat content whereas the formation of oxidized cholesterol products is further increased by roasting (Echarte et al., 2001). Similar results were found in a Finnish prospective cohort study from the Kuopio Ischemic Heart Disease Risk Factor Study (Virtanen et al., 2009) 


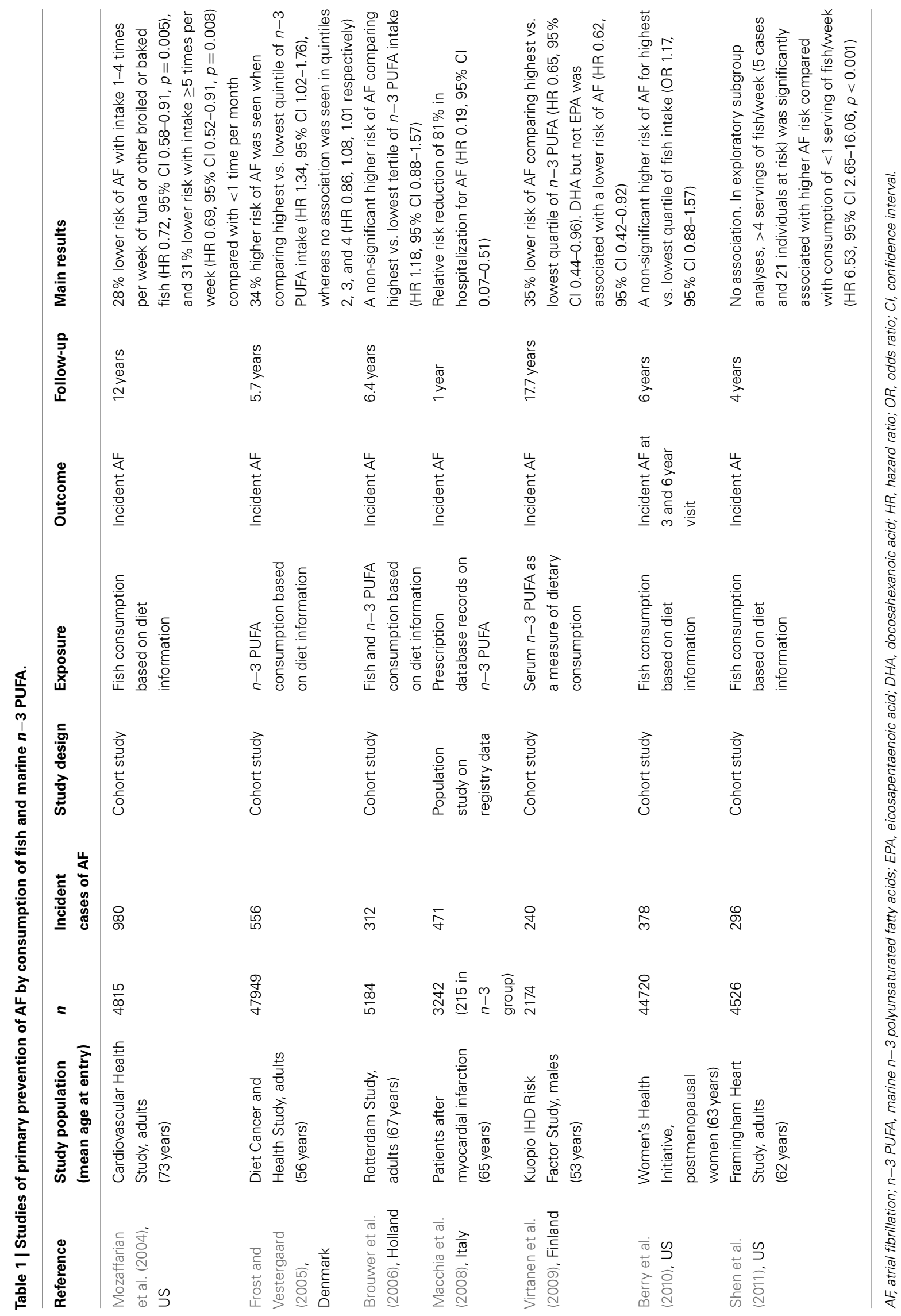


where a total of 240 incident cases of AF occurred during 18 years of follow-up among 2174 men aged between 42 and 60 years. The highest quartile of total $n-3$ PUFA content in serum at baseline was associated with a $35 \%$ lower risk of AF compared to the lowest quartile (HR 0.65, 95\% CI 0.44-0.96, $p$ for trend =0.07). Serum DHA (but not EPA) was associated with the risk of AF, with a $38 \%$ lower risk of AF comparing the highest and lowest quartiles (HR $0.62,95 \%$ CI $0.42-0.92$, $p$ for trend $=0.02$ ). This inverse association has, however, not been found in other cohort studies (Frost and Vestergaard, 2005; Brouwer et al., 2006; Berry et al., 2010; Shen et al., 2011). Thus, in the study by Frost and Vestergaard (2005), there was a significantly higher risk of AF when comparing the highest vs. the lowest quintile of $n-3$ PUFA consumption. In an exploratory analysis in the study by Shen et al. (2011), the highest intake of dark (fatty) fish was also associated with a higher risk of AF. Overall, these studies have shown no clear association between higher fish or $n-3$ PUFA intake and incident AF. Finally, an Italian registry-based study on prescription of fish oil supplements for patients discharged after a myocardial infarction showed that significantly fewer had AF within the next year in the group prescribed fish oil supplements (Macchia et al., 2008).

Differences exist between the studies that may explain some of these contradictory results. The participants in the study by Mozaffarian et al. (2004) were older compared to the other studies, and the effects of fish intake may be more effective in this age category. First, the background diet in the population as well as the available fish products differ between studies Next, the causes of AF may differ between the study populations, and therefore the susceptibility to the effects of marine $n-3$ PUFA may differ. Also, patients may be motivated to a higher fish intake, for instance because of symptomatic coronary heart disease, and this would select persons who have higher morbidity and a higher a priori risk of AF, which in turn would bias the results in the direction of a detrimental effect of intake. It may also be that the effect, if any, of fish and fish oils is not simply a linear doseresponse but may instead have a different association such as threshold effect or even a U- or J-shaped effect with detrimental impacts at both very low and very high levels. If a threshold effect is indeed present, establishing this would be very much dependent on the distribution of intake in the study population. Larger studies would be helpful in clarifying these issues, e.g., by using continuous exposure measures such as cubic splines. Although primary prevention of AF is of major importance, it is not likely that it would be feasible to conduct a RCT since this would require a very large study. For this reason, exploring a possible effect on primary prevention will be much dependent on further epidemiological studies.

\section{PREVENTION OF RECURRENCE OF AF AFTER CARDIOVERSION}

Although primary prevention of AF is very important for reducing the AF burden in a population, it is more feasible to motivate patients for secondary prevention of AF, where there is a need for safe and well-tolerated treatment options. As this setting is different, with varying degrees of electrical and structural remodeling ongoing or finalized, an effect of treatment may work by different mechanisms compared to the setting of primary prevention where effects on cardiovascular risk factors such as hypertension may be more predominant. For this reason, it is important to consider the type of patients when comparing results from different studies.

Recently, a number of RCT has been reported (Table 2), of which two showed a beneficial effect of treatment with marine n-3 PUFA (Nodari et al., 2011; Kumar et al., 2012), whereas this was not found in three other studies (Kowey et al., 2010; Bianconi et al., 2011; Ozaydin et al., 2011).

Thus, in a study by Nodari et al. (2011), 199 patients with persistent AF who were treated with amiodarone and a reninangiotensin-aldosterone system inhibitor were randomized to $1.7 \mathrm{~g} n-3$ PUFA/day or placebo 4 weeks before electrical cardioversion. The primary endpoint was maintenance of sinus rhythm at 1-year follow-up, and of the $n-3$ PUFA-treated patients, $62 \%$ were still in sinus rhythm compared to $36 \%$ in the placebo group $(p<0.0001)$.

Also in favor of a beneficial effect of $n-3$ PUFA is a recent study by Kumar et al. (2012) involving 178 patients with persistent AF who were randomized to $1.8 \mathrm{~g} n-3$ PUFA/day or placebo for at least 1 month prior to electrical cardioversion. Primary endpoint was recurrence of persistent AF defined as AF documented for at least 1 week. At 90 days, $39 \%$ in the $n-3$ PUFA group had AF recurrence compared to $78 \%$ of controls (HR 0.38, 95\% CI 0.27$0.56, p<0.001)$. At 1 year, $67 \%$ of $n-3$ PUFA-treated patients and $90 \%$ of controls had persistent AF $(p<0.001)$. Extending the findings by Nodari et al., $n-3$ PUFA were associated with a significant reduction in AF recurrence with or without concurrent anti-arrhythmic drugs. An additional finding was that increasing levels of DHA as a percentage of fatty acids in the phospholipid fraction of serum predicted a lower risk of AF recurrence (HR 0.59, 95\% CI $0.42-0.83, p=0.003)$, whereas this was not statistically significant for EPA (HR 0.9, 95\% CI 0.8-1.1, $p=0.08$ ).

Contrary to these findings, no effect of treatment was found in a large study by Kowey et al. (2010) in patients with paroxysmal AF $(n=542)$ or persistent AF $(n=121)$ without structural heart disease. The patients were in sinus rhythm at baseline and randomized to $6.7 \mathrm{~g} n-3$ PUFA/day for 7 days and $4 \mathrm{~g} /$ day thereafter. There was no difference between treatment groups for recurrence of symptomatic AF in the paroxysmal stratum (HR 1.15, 95\% CI $0.90-1.46, p=0.26$ ), in the persistent stratum (HR 1.64, 95\% CI $0.92-2.92, p=0.09$ ), or in both strata combined (HR 1.22, 95\% CI $0.98-1.52, p=0.08)$. Importantly, almost half of AF recurrences occurred within 2 weeks of $n-3$ PUFA supplementation. In comparison with the studies by Nodari et al. (2011) and Kumar et al. (2012), it can be argued that the effect of $n-3$ PUFA may not be fully penetrant this early, since it takes about 30 days for full incorporation of $n-3$ PUFA into atrial myocytes (Owen et al., 2004; Metcalf et al., 2007). Other differences were that patients were not on anti-arrhythmic drug treatment, had no structural heart disease, and predominantly had paroxysmal AF, and therefore a lesser degree of atrial remodeling.

Likewise, no effect was found by Bianconi et al. (2011) in a study of 204 patients with persistent AF randomized to $2.6 \mathrm{~g} n-3$ PUFA/day or placebo starting $\geq 7$ days (mean 3 weeks) prior to cardioversion and $2 \mathrm{~g} /$ day thereafter. The primary endpoint was AF recurrence which occurred in 59\% of the $n-3$ PUFA-treated patients and in $51 \%$ of controls $(p=0.28)$. Compared to the studies by Nodari et al. (2011) and Kumar et al. (2012), a lack of effect 


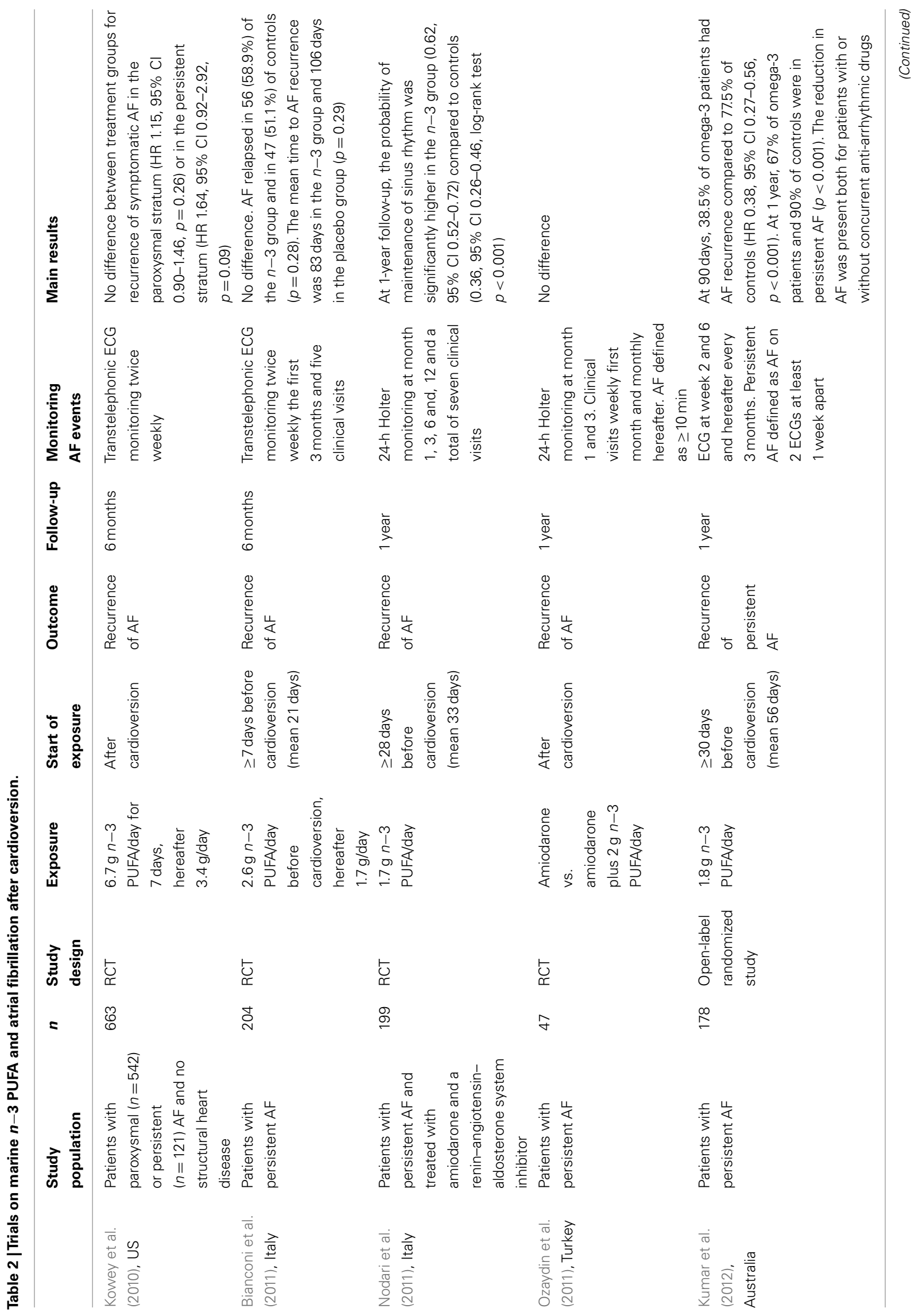




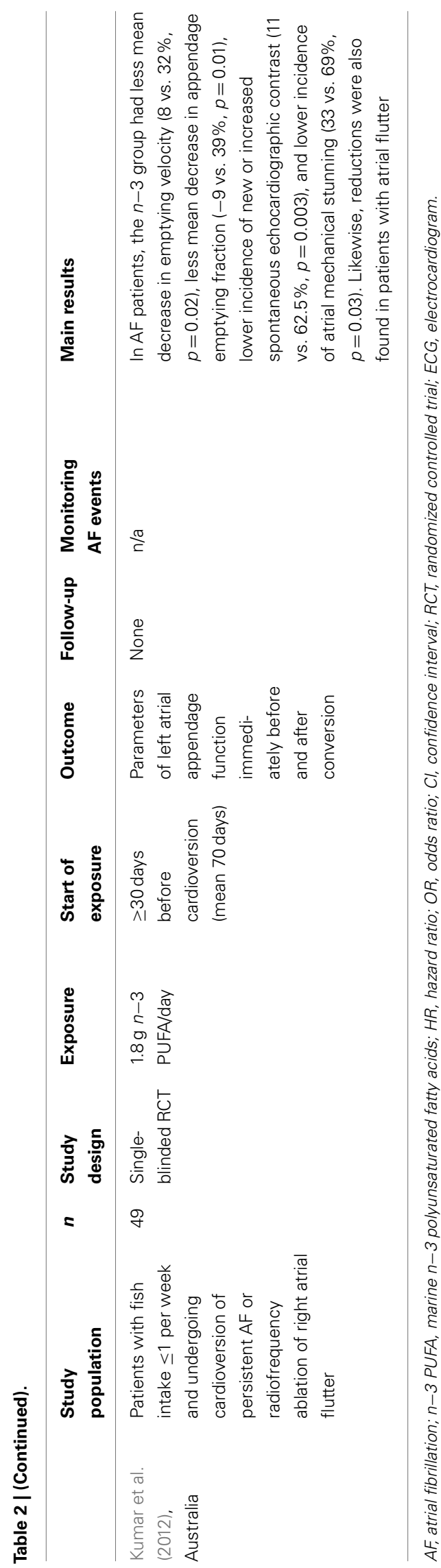

of $n-3$ PUFA supplementation may be explained by the fact that the majority of patients had been treated for less than 30 days prior to cardioversion. Differences in the background diet may also be a contributory factor, since the DHA concentration in blood only increased by $25 \%$ which is lower than 1.8 -fold in the study by Kumar et al. (2012), suggesting a smaller difference between the intervention group and the placebo group. Finally, a small study by Ozaydin et al. (2011) with $n-3$ PUFA started after cardioversion also found no difference between groups.

In support of an effect of $n-3$ PUFA supplementation in patients with persistent AF are the results of a study on 49 patients concerning atrial mechanical function after reversal to sinus rhythm (Kumar et al., 2011c). After reversion of persistent AF (external or internal cardioversion) or persistent right atrial flutter (radiofrequency ablation) to sinus rhythm, parameters of left atrial appendage function were compared immediately before and after reversion. Consumption of $1.8 \mathrm{~g} n-3$ PUFA/day for $\geq 1$ month prior to reversion was shown to attenuate atrial mechanical stunning after reversion of AF and right atrial flutter to sinus rhythm.

At this point, more studies are needed to establish whether the potential beneficial effect on persistent AF observed in some studies is valid. It seems important to consider $\geq 30$ days pre-treatment with $n-3$ PUFA to allow for incorporation in cell membranes. Notably, an effect on top of treatment with both amiodarone and a renin-angiotensin-aldosterone system inhibitor has been reported.

\section{PREVENTION OF POST-OPERATIVE AF}

Patients undergoing heart surgery such as coronary artery bypass grafting (CABG) often develop $\mathrm{AF}$ after the operation. In this setting, $\mathrm{AF}$ is induced by the intervention, and the mechanism is probably mediated by the highly inflammatory state following surgery.

A number of RCT have examined the effects of $n-3$ PUFA in patients undergoing heart surgery, especially CABG (Table 3 ). Promising results were initially published by Calo et al. (2005) in a study of 160 patients undergoing CABG. Patients were randomized to $1.7 \mathrm{~g} n-3$ PUFA/day or placebo $\geq 5$ days prior to surgery and were followed until discharge. The primary endpoint was the development of post-operative $\mathrm{AF}$, which was seen in only $15 \%$ in the $n-3$ PUFA group compared to $33 \%$ of controls $(p=0.013)$. In addition, the $n-3$ PUFA group were hospitalized for significantly fewer days than the controls (7.3 vs. 8.2 days, $p=0.017$ ).

Some support for a beneficial effect of $n-3$ PUFA was also reported by Sorice et al. (2011) in a study of 201 patients randomized to on-pump or off-pump CABG and supplement with $1.7 \mathrm{~g} n-3$ PUFA/day or placebo. A significant reduction in postoperative $\mathrm{AF}$ was found in on-pump CABG patients treated with $n-3$ PUFA ( 11.7 vs. $31.6 \%$, OR $0.28, p=0.01$ ), whereas no difference was seen for $n-3$ PUFA in off-pump CABG patients (11.1 vs. $12.5 \%$, OR 0.87, $p=0.84$ ). Also, Farquharson et al. (2011) studied 194 patients undergoing CABG or valve operating procedures who were randomized to $4.5 \mathrm{~g} n-3 \mathrm{PUFA} /$ day or placebo starting 3 weeks prior to surgery. There was a $37 \%$ reduction of post-operative $\mathrm{AF}$ in the intervention group, however not significant (OR 0.63, 95\% CI 0.35-1.11), and a non-significant delay in 


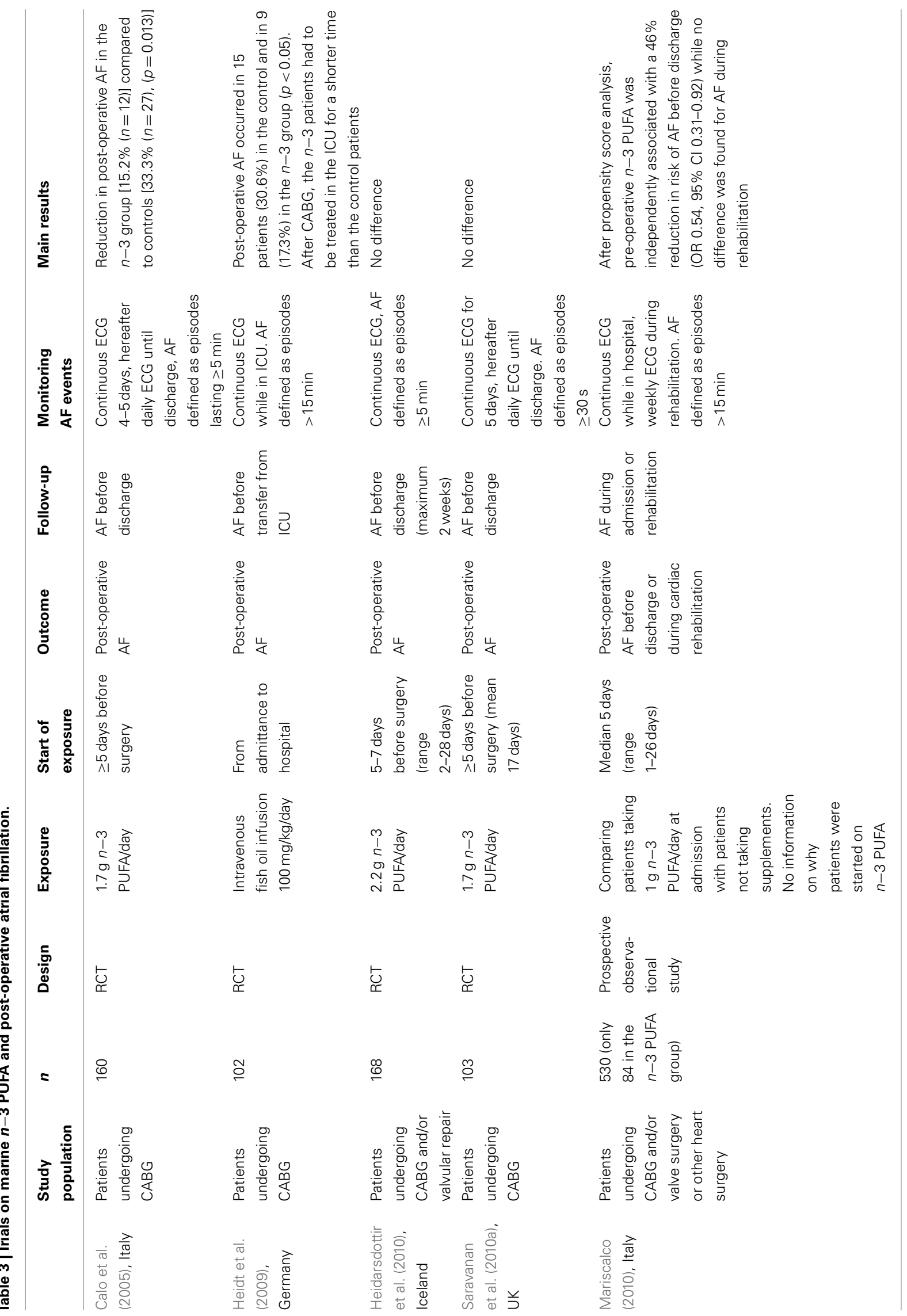




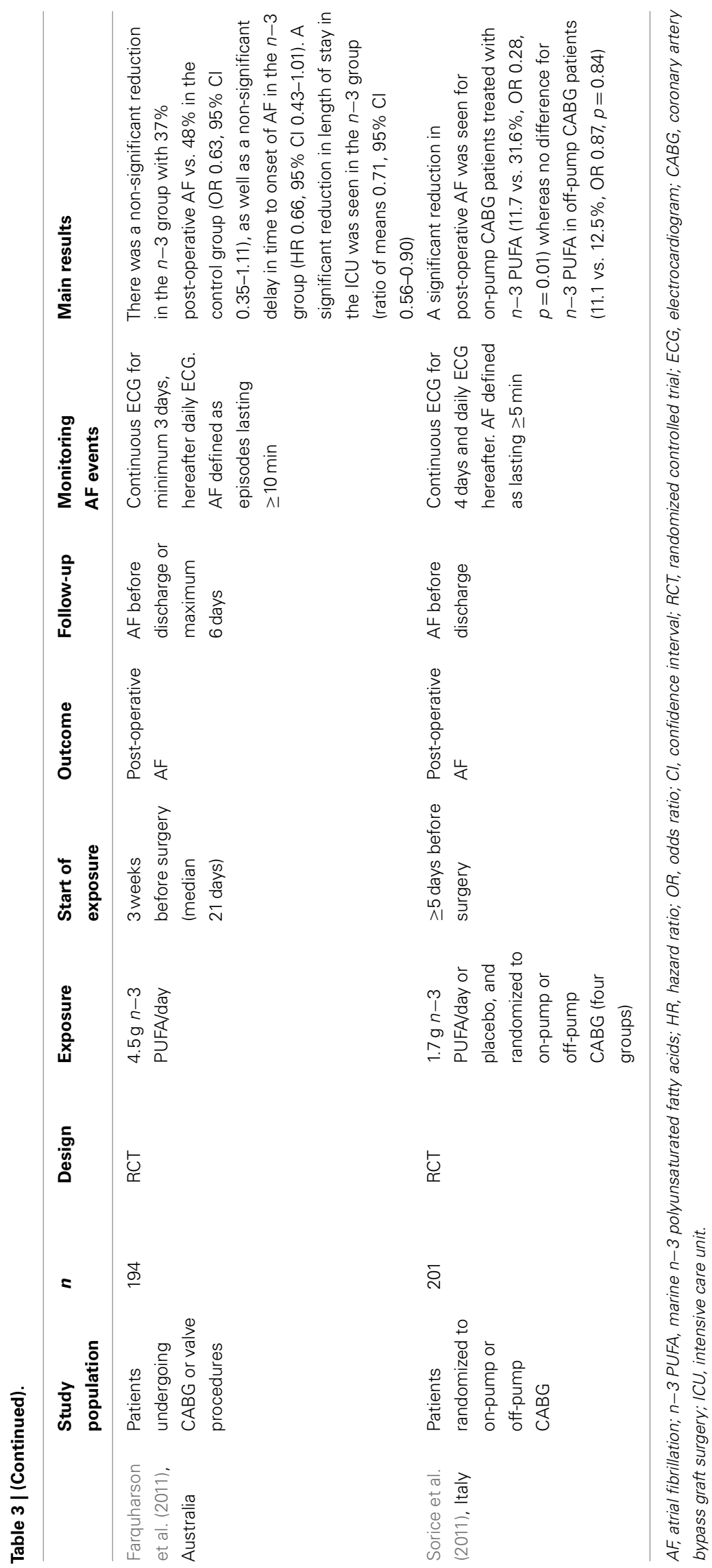




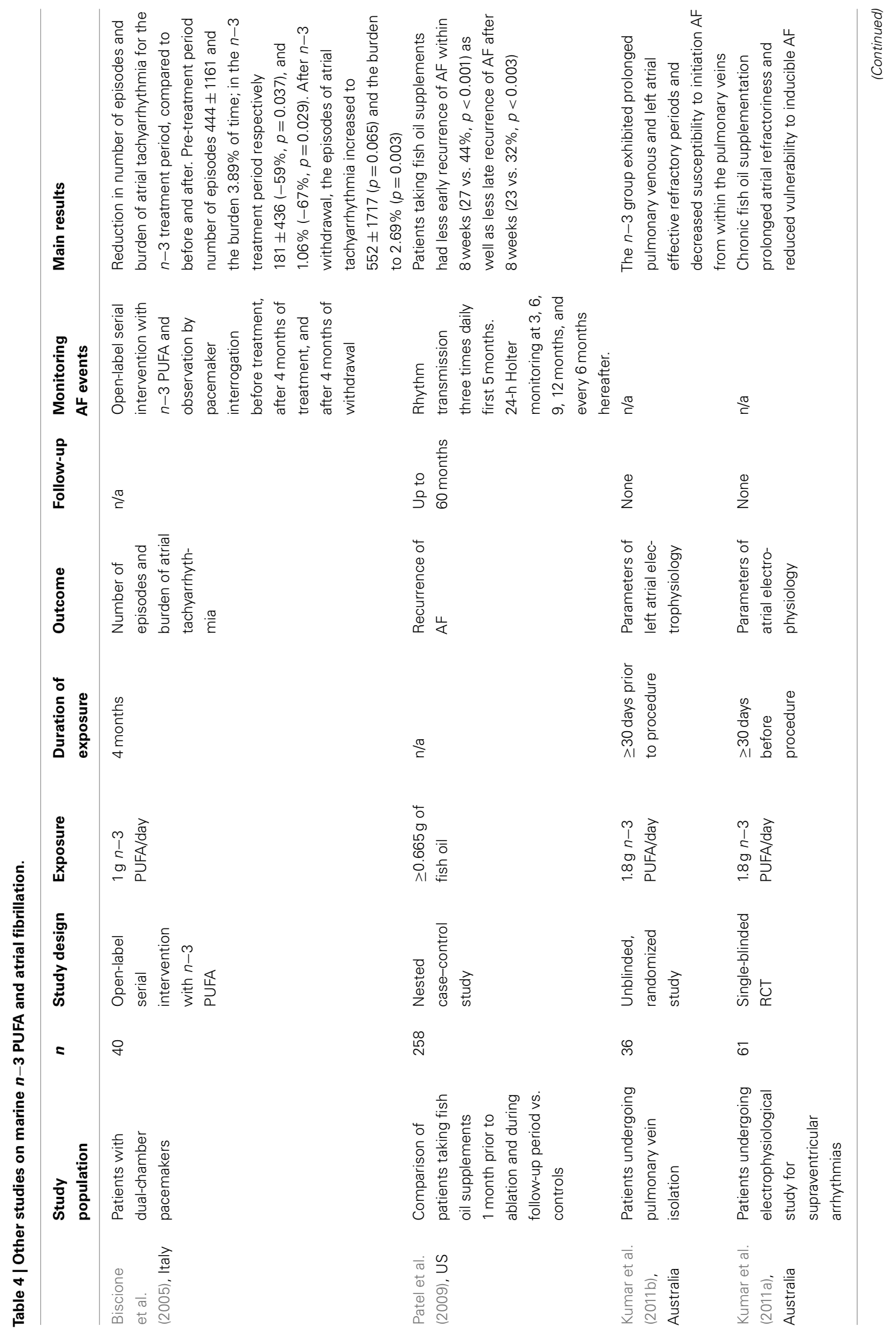




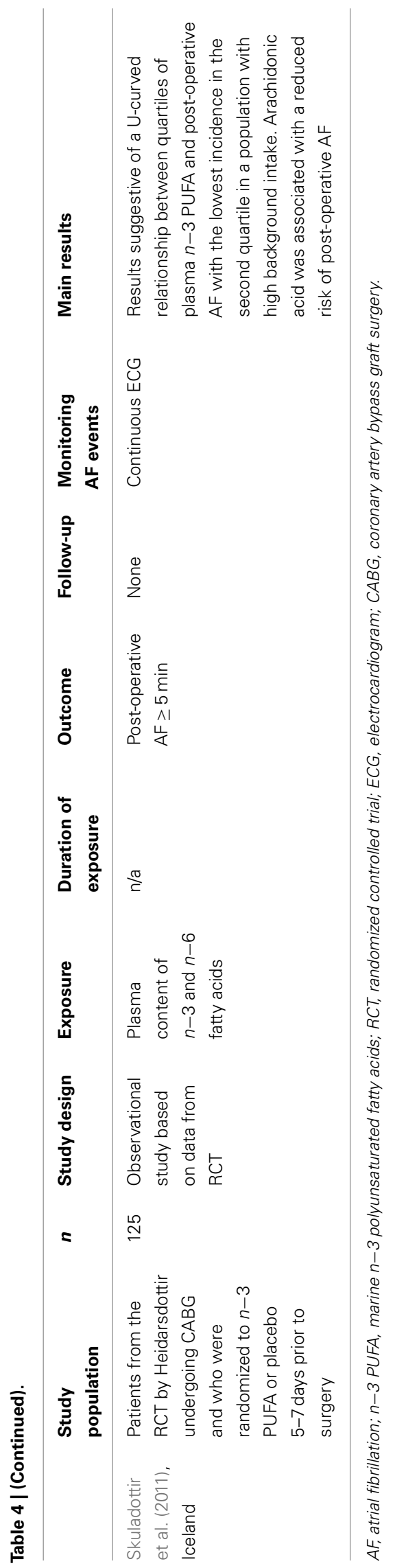

time to onset of AF (HR $0.66,95 \%$ CI $0.43-1.01$ ). Length of stay in the intensive care unit (ICU) was significantly decreased in the $n-3$ PUFA group (ratio of means $0.71,95 \%$ CI $0.56-0.90$ ).

In opposition to these findings, two RCT showed no effect of $n-3$ PUFA on the incidence of post-operative AF. Thus, in a study by Saravanan et al. (2010a) of 108 patients scheduled for CABG and randomized to $1.7 \mathrm{~g} n-3$ PUFA/day or placebo and followed for 5 days with continuous ECG, no difference in AF was found between groups. Compared to the above studies, the definition of AF was duration $\geq 30 \mathrm{~s}$ instead of $>5$ to $>15 \mathrm{~min}$, which explains a high rate of AF. The other study by Heidarsdottir et al. (2010) in Iceland with 168 patients treated with CABG and/or valve surgery and randomized to $2.2 \mathrm{~g} n-3$ PUFA/day or placebo found no difference between groups. However, the background diet in this population was rich in cod liver oil and $n-3$ PUFA supplements, and the percentage of $n-3$ PUFA in plasma phospholipids was about three times higher than that reported in an Italian study (Abbatecola et al., 2009) which may be comparable to the study by Calo et al. (2005). For this reason, the high background intake of $n-3$ PUFA and a relatively small increase in the intervention group may explain why no effect of marine $n-3$ PUFA could be demonstrated.

In a study of the acute effects of $n-3$ PUFA, 102 patients undergoing $\mathrm{CABG}$ were randomized to intravenous infusion $100 \mathrm{mg} / \mathrm{kg} /$ day of fish oil or placebo (Heidt et al., 2009). Infusion was started at admission and continued until the end of followup at transfer from the ICU. Post-operative AF occurred in 15 patients $(31 \%)$ in the control group but only in $9(17 \%)$ in the $n-3$ PUFA group $(p<0.05)$. The $n-3$ PUFA-treated patients also had a significantly shorter stay in the ICU.

The available data seem fairly promising, but more studies are needed to establish whether $n-3$ PUFA supplementation is beneficial in patients undergoing heart surgery. Possible effects in this special setting of heart surgery with a high inflammatory response may not be directly comparative to other settings such as primary or secondary prevention. However, prevention of post-operative AF by a safe and well-tolerated treatment would be a compelling treatment option as this would reduce morbidity in these patients as well as the time spent in hospital. For future studies, a longer pre-operative treatment for at least 1 month may be considered to allow for a higher incorporation of $n-3$ PUFA into cardiac cell membranes.

\section{n-3 PUFA AND AF PATIENTS WITH PACEMAKERS}

In a study of AF patients with dual-chamber pacemakers, Biscione et al. (2005) conducted an open-label serial intervention with $1 \mathrm{~g} n-3$ PUFA/day and observation by pacemaker interrogation before treatment, after 4 months of treatment, and after 4 months of withdrawal (Table 4). A significant reduction in number of episodes and burden of atrial tachyarrhythmia was found during $n-3$ PUFA treatment. These interesting findings, however, need to be confirmed in other studies.

\section{n-3 PUFA AND RADIO FREQUENCY ABLATION}

A lower risk of early recurrence ( $\leq 8$ weeks) and procedural failure ( $>8$ weeks) was reported in a nested case-control study from a cohort of patients treated with pulmonary vein antrum ablation 
for AF (Patel et al., 2009; Table 4). Among the 1500 treated patients, patients consuming $\geq 665 \mathrm{mg}$ of fish oil were matched with controls (129 in each group). Also, in an unblinded, randomized study on patients undergoing pulmonary vein isolation, patients randomized to $1.8 \mathrm{~g} n-3$ PUFA/day had prolonged pulmonary venous and left atrial effective refractory periods and decreased susceptibility to initiation of AF from within the pulmonary veins (Kumar et al., 2011b). In comparison, the effects of $n-3$ PUFA on electrophysiologic parameters were studied in a single-blinded study of patients with supraventricular tachycardia but without AF or structural heart disease (Kumar et al., 2011a). The participants had a low fish intake and were randomized to $1.8 \mathrm{~g} n-3$ PUFA/day for at least 1 month, and effects of $n-3$ PUFA treatment included lengthening of atrial refractory period, less inducible, and shorter duration of AF.

\section{WHY ARE RESULTS ON MARINE $\boldsymbol{n}-3$ PUFA AND AF INCONSISTENT?}

One explanation for the mixed results of the effects of $n-3$ PUFA could be that the effect is not simply a linear dose-response but may be, e.g., a threshold effect between very low intake and nor$\mathrm{mal} /$ high intake or even a J- or U-shaped effect where very low and very high levels are detrimental, as suggested in a study on plasma content of $n-3$ PUFA and post-operative AF (Skuladottir et al., 2011). Also, as AF is a disease that may be caused by a wide range of different factors and in turn, $n-3$ PUFA also has been ascribed a long list of possible mechanisms of action on AF, the association of effect is likely to vary between populations according to background intake of $n-3$ PUFA, the overall health status of patients, and the type and duration of AF.

When comparing information from observational studies and intervention studies, one must keep in mind that there are important differences in the amounts of $n-3$ PUFA investigated in these two types of designs. Observational studies often compare persons with higher intakes against persons with very low intakes, whereas in interventional studies a high dose is usually investigated against a control group with an average intake that represents the whole study population. For this reason, for example if there is a threshold effect of $n-3$ PUFA between the lowest quartile of fish intake, this may not be tested appropriately in a standard RCT. Likewise, if there is an upper limit to the beneficial effect of $n-3$ PUFA, this could also be a problem in RCT where some persons are likely to have a high background intake of fish supplemented with high doses in capsules.

The concomitant treatment with other drugs that may potentially work by some of the same mechanisms as $n-3$ PUFA may also be relevant. Thus, in a recent study in patients with a history of myocardial infarction, low-dose supplementation with $n-3$ fatty acids was associated with a reduction in major cardiovascular events in patients not treated with statins, whereas in statin users no difference was found (Eussen et al., 2012). Medications that affect atrial remodeling by reducing atrial fibrosis and

\section{REFERENCES}

Abbatecola, A. M., Cherubini, A., Guralnik, J. M., Andres Lacueva, C., Ruggiero, C., Maggio, M., Bandinelli, S., Paolisso, G., and Ferrucci, L. (2009). Plasma polyunsaturated fatty acids and age-related physical performance decline. Rejuvenation Res. 12, 25-32.

Allessie, M., Ausma, J., and Schotten, U. (2002). Electrical, contractile and structural remodeling during

also reduce AF may include statins and inhibitors of the reninangiotensin-aldosterone system (Nattel et al., 2008). It is possible that the potential effect of additional treatment with $n-3$ PUFA in these patients may be less.

A problem when studying AF is that while some patients are bothered by symptoms of AF and therefore more likely to be diagnosed, a large proportion are unaffected and only diagnosed by chance or not at all. Also, even symptomatic patients have asymptomatic episodes of AF. Thus, the proportions of symptomatic and asymptomatic patients vary between studies according to the background population, and recurrence rates during followup are increased depending on the completeness of heart rhythm monitoring during follow-up (e.g., continuous or ambulant ECG) which will have a major effect on the outcome and may explain the diverging results obtained.

\section{KEY QUESTIONS FOR FURTHER RESEARCH}

Based on the available studies in regard to AF after cardioversion or heart surgery, it seems reasonable to consider a preprocedure treatment of at least 30 days for interventional studies examining the effect of dietary $n-3$ PUFA. Future studies are needed to clarify whether a relation between dietary intake of $n-3$ PUFA and AF is linear, U- or J-shaped or has a threshold above which no further effect is obtained. More knowledge on this has great implications both for comparing studies in populations with different background dietary intake and dose of exposure, and for establishing the optimal dosage. It is likely that some conditions will have a higher potential for an effect of $n-3$ PUFA, and identifying these conditions would be important for patient treatment. Experimental data suggest differences between the acute effects of intravenous infusion of $n-3$ PUFA and the chronic effects of dietary $n-3$ PUFA incorporated into cell membranes, and therefore studies should be interpreted according to the type of exposure. Finally, the endpoint in most studies is first episode of AF. Although this is a measurable endpoint, it may well be that if only a small proportion can achieve freedom from AF, patients with AF would still be interested in a treatment option that may reduce the number and severity of episodes and burden of time in AF, as exemplified by Biscione et al. (2005).

\section{CONCLUSION}

There is growing evidence for an effect of marine $n-3$ PUFA in prevention and treatment of AF. However, further studies are needed to establish which patients are more likely to benefit from $n-3$ PUFA, timing of treatment, and dosages.

\section{ACKNOWLEDGMENTS}

This study was supported by a research grant from the Danish Council for Strategic Research (grant number 09-066965) and a grant from The Obel Family Foundation.

atrial fibrillation. Cardiovasc. Res. 54, 230-246.

Berry, J. D., Prineas, R. J., van Horn, L. Passman, R., Larson, J., Goldberger,

J., Snetselaar, L., Tinker, L., Liu,

K., and Lloyd-Jones, D. M. (2010).
Dietary fish intake and incident atrial fibrillation (from the women's health initiative). Am. J. Cardiol. 105, 844-848.

Bianconi, L., Calo, L., Mennuni, M., Santini, L., Morosetti, P., Azzolini, P., 
Barbato, G., Biscione, F., Romano, P., and Santini, M. (2011). N-3 polyunsaturated fatty acids for the prevention of arrhythmia recurrence after electrical cardioversion of chronic persistent atrial fibrillation: a randomized, double-blind, multicentre study. Europace 13, 174-181.

Billman, G. E., Kang, J. X., and Leaf, A. (1999). Prevention of sudden cardiac death by dietary pure omega-3 polyunsaturated fatty acids in dogs. Circulation 99, 2452-2457.

Billman, G. E., Nishijima, Y., Belevych, A. E., Terentyev, D., Xu, Y., Haizlip, K. M., Monasky, M. M., Hiranandani, N, Harris, W. S., Gyorke, S., Carnes, C. A., and Janssen, P. M. L. (2010). Effects of dietary omega-3 fatty acids on ventricular function in dogs with healed myocardial infarctions: in vivo and in vitro studies. Am. J. Physiol. Heart Circ. Physiol. 298, H1219-H1228.

Biscione, F., Totteri, A., De Vita, A., Lo Bianco, F., and Altamura, G. (2005). Effect of omega-3 fatty acids on the prevention of atrial arrhythmias. Ital. Heart Suppl. J. 6, 53-59.

Bjerregaard, L. J., Joensen, A. M., Dethlefsen, C., Jensen, M. K., Johnsen, S. P., Tjonneland, A., Rasmussen, L. H., Overvad, K., and Schmidt, E. B. (2010). Fish intake and acute coronary syndrome. Eur. Heart J. 31, 29-34.

Brouwer, I. A., Heeringa, J., Geleijnse, J. M., Zock, P. L., and Witteman, J. C. (2006). Intake of very long-chain n3 fatty acids from fish and incidence of atrial fibrillation. The rotterdam study. Am. Heart J. 151, 857-862.

Burdge, G. C., and Calder, P. C. (2005). Conversion of alpha-linolenic acid to longer-chain polyunsaturated fatty acids in human adults. Reprod. Nutr. Dev. 45, 581-597.

Calder, P. C. (2006). N-3 polyunsaturated fatty acids, inflammation, and inflammatory diseases. Am. Clin. J. Nutr. 83, 1505S-1519S.

Calder, P. C. (2012). Mechanisms of action of (n-3) fatty acids. Nutr. J. 142, 592S-5929S.

Calo, L., Bianconi, L., Colivicchi, F., Lamberti, F., Loricchio, M. L., de Ruvo, E., Meo, A., Pandozi, C., Staibano, M., and Santini, M. (2005). $\mathrm{N}-3$ fatty acids for the prevention of atrial fibrillation after coronary artery bypass surgery: a randomized, controlled trial. Am. J. Coll. Cardiol. 45, 1723-1728.

da Cunha, D. N., Hamlin, R. L., Billman, G. E., and Carnes, C. A. (2007). N3 (omega-3) polyunsaturated fatty acids prevent acute atrial electrophysiological remodeling. $\mathrm{Br}$. J. Pharmacol. 150, 281-285.

De Caterina, R. (2011). N-3 fatty acids in cardiovascular disease. Engl. N. J. Med. 364, 2439-2450.

Den Ruijter, H. M., Berecki, G., Opthof, T., Verkerk, A. O., Zock, P. L., and Coronel, R. (2007). Pro- and antiarrhythmic properties of a diet rich in fish oil. Cardiovasc. Res. 73, 316-325.

Den Ruijter, H. M., and Coronel, R. (2009). The response to fish oil in patients with heart disease depends on the predominant arrhythmia mechanism. Cardiovasc. Drugs Ther. 23, 333-334.

Dun, W., and Boyden, P. A. (2009). Aged atria: electrical remodeling conducive to atrial fibrillation. J. Interv. Card. Electrophysiol. 25, 9-18.

Dyerberg, J., Bang, H. O., Stoffersen, E., Moncada, S., and Vane, J. R. (1978). Eicosapentaenoic acid and prevention of thrombosis and atherosclerosis? Lancet 2, 117-119.

Echarte, M., Zulet, M. A., and Astiasaran, I. (2001). Oxidation process affecting fatty acids and cholesterol in fried and roasted salmon. Agric. J. Food Chem. 49, 5662-5667.

Eussen, S. R., Geleijnse, J. M., Giltay, E. J., Rompelberg, C. J., Klungel, O. H., and Kromhout, D. (2012). Effects of n-3 fatty acids on major cardiovascular events in statin users and nonusers with a history of myocardial infarction. Eur. Heart J.

Farquharson, A. L., Metcalf, R. G., Sanders, P., Stuklis, R., Edwards, J. R., Gibson, R. A., Cleland, L. G., Sullivan, T. R., James, M. J., and Young, G. D. (2011). Effect of dietary fish oil on atrial fibrillation after cardiac surgery. Am. Cardiol. J. 108, 851-856.

Frost, L., and Vestergaard, P. (2005). n-3 Fatty acids consumed from fish and risk of atrial fibrillation or flutter: the danish diet, cancer, and health study. Am. Clin. J. Nutr. 81, 50-54.

Heidarsdottir, R., Arnar, D. O., Skuladottir, G. V., Torfason, B., Edvardsson, V., Gottskalksson, G., Palsson, R., and Indridason, O. S. (2010). Does treatment with n-3 polyunsaturated fatty acids prevent atrial fibrillation after open heart surgery? Europace 12, 356-363.

Heidt, M. C., Vician, M., Stracke, S. K. Stadlbauer, T., Grebe, M. T., Boening, A., Vogt, P. R., and Erdogan, A. (2009). Beneficial effects of intravenously administered N-3 fatty acids for the prevention of atrial fibrillation after coronary artery bypass surgery: a prospective randomized study. Thorac. Cardiovasc. Surg. 57, 276-280.

Jahangiri, A., Leifert, W. R., Patten, G. S., and McMurchie, E. J. (2000). Termination of asynchronous contractile activity in rat atrial myocytes by $\mathrm{n}$ 3 polyunsaturated fatty acids. Mol. Cell. Biochem. 206, 33-41.

Kang, J. X., and Leaf, A. (1996). Protective effects of free polyunsaturated fatty acids on arrhythmias induced by lysophosphatidylcholine or palmitoylcarnitine in neonatal rat cardiac myocytes. Eur. Pharmacol. J. 297, 97-106.

Kitamura, K., Shibata, R., Tsuji, Y., Shimano, M., Inden, Y., and Murohara, T. (2011). Eicosapentaenoic acid prevents atrial fibrillation associated with heart failure in a rabbit model. Am. Physiol. J. Heart Circ Physiol. 300, H1814-H1821.

Kowey, P. R., Reiffel, J. A., Ellenbogen, K. A., Naccarelli, G. V., and Pratt, C. M. (2010). Efficacy and safety of prescription omega-3 fatty acids for the prevention of recurrent symptomatic atrial fibrillation: a randomized controlled trial. JAMA 304, 2363-2372.

Kumar, S., Sutherland, F., Morton, J. B. Lee, G., Morgan, J., Wong, J., Eccleston, D. E., Voukelatos, J., Garg, M. L., and Sparks, P. B. (2012). Longterm omega-3 polyunsaturated fatty acid supplementation reduces the recurrence of persistent atrial fibrillation after electrical cardioversion. Heart Rhythm 9, 483-491.

Kumar, S., Sutherland, F., Rosso, R., Teh, A. W., Lee, G., Heck, P. M., Feldman, A., Medi, C., Watt, S., Garg, M. L., and Sparks, P. B. (2011a) Effects of chronic omega-3 polyunsaturated fatty acid supplementation on human atrial electrophysiology. Heart Rhythm 8, 562-568.

Kumar, S., Sutherland, F., Teh, A. W. Heck, P. M., Lee, G., Garg, M. L., and Sparks, P. B. (2011b). Effects of chronic omega-3 polyunsaturated fatty acid supplementation on human pulmonary vein and left atrial electrophysiology in paroxysmal atrial fibrillation. Am. Cardiol. J. 108, 531-535.

Kumar, S., Sutherland, F., Wheeler, M. Heck, P. M., Lee, G., Teh, A. W., Garg, M. L., Morgan, J. G., and Sparks, P. B. (2011c). Effects of chronic omega-3 polyunsaturated fatty acid supplementation on human atrial mechanical function after reversion of atria arrhythmias to sinus rhythm: reversal of tachycardia-mediated atrial cardiomyopathy with fish oils. Heart Rhythm 8, 643-649.
Laurent, G., Moe, G., Hu, X., Holub, B., Leong-Poi, H., Trogadis, J., Connelly, K., Courtman, D., Strauss, B. H., and Dorian, P. (2008). Long chain n-3 polyunsaturated fatty acids reduce atrial vulnerability in a novel canine pacing model. Cardiovasc. Res. 77, 89-97.

Leaf, A., Xiao, Y. F., Kang, J. X., and Billman, G. E. (2005). Membrane effects of the n-3 fish oil fatty acids, which prevent fatal ventricular arrhythmias. Membr. J. Biol. 206, 129-139.

Macchia, A., Monte, S., Pellegrini, F., Romero, M., Ferrante, D., Doval, H., D’Ettorre, A., Maggioni, A. P., and Tognoni, G. (2008). Omega-3 fatty acid supplementation reduces one-year risk of atrial fibrillation in patients hospitalized with myocardial infarction. Eur. Clin. J. Pharmacol. 64, 627-634.

Mariscalco, G., Sarzi Braga, S., Banach, M., Borsani, P., Bruno, V. D. Napoleone, M., Vitale, C., Piffaretti, G., Pedretti, R. F., and Sala, A (2010). Preoperative n-3 polyunsatured fatty acids are associated with a decrease in the incidence of early atrial fibrillation following cardiac surgery. Angiology 61, 643-650.

Mayyas, F., Sakurai, S., Ram, R., Rennison, J. H., Hwang, E. S., Castel, L. Lovano, B., Brennan, M. L., Bibus, D., Lands, B., Barnard, J., Chung, M. K., and Van Wagoner, D. R. (2011). Dietary omega3 fatty acids modulate the substrate for post-operative atrial fibrillation in a canine cardiac surgery model. Cardiovasc. Res. 89, 852-861.

McLennan, P. L. (1993). Relative effects of dietary saturated, monounsaturated, and polyunsaturated fatty acids on cardiac arrhythmias in rats. Am. Clin. J. Nutr. 57, 207-212.

McLennan, P. L., Bridle, T. M., Abeywardena, M. Y., and Charnock, J. S. (1993). Comparative efficacy of n-3 and n-6 polyunsaturated fatty acids in modulating ventricular fibrillation threshold in marmoset monkeys. Am. Clin. J. Nutr. 58, 666-669.

Metcalf, R. G., James, M. J., Gibson, R. A., Edwards, J. R., Stubberfield, J., Stuklis, R., Roberts-Thomson, K., Young, G. D., and Cleland, L. G. (2007). Effects of fish-oil supplementation on myocardial fatty acids in humans. Am. Clin. J. Nutr. 85, 1222-1228.

Mozaffarian, D., Psaty, B. M., Rimm, E. B., Lemaitre, R. N., Burke, G. L. Lyles, M. F., Lefkowitz, D., and Siscovick, D. S. (2004). Fish intake and risk of incident atrial fibrillation. Circulation 110, 368-373. 
Mozaffarian, D., and Wu, J. H. (2011). Omega-3 fatty acids and cardiovascular disease: effects on risk factors, molecular pathways, and clinical events. Am. J. Coll. Cardiol. 58, 2047-2067.

Nattel, S., Burstein, B., and Dobrev, D. (2008). Atrial remodeling and atrial fibrillation: mechanisms and implications. Circ. Arrhythm. Electrophysiol. 1, 62-73.

Nattel, S., and Van Wagoner, D. R. (2011). Atrial fibrillation: therapy with omega- 3 fatty acids-is the case closed? Nat. Rev. Cardiol. 8, 126-128.

Ninio, D. M., Murphy, K. J., Howe, P. R., and Saint, D. A. (2005). Dietary fish oil protects against stretch-induced vulnerability to atrial fibrillation in a rabbit model. Cardiovasc. J. Electrophysiol. 16, 1189-1194.

Nodari, S., Triggiani, M., Campia, U., Manerba, A., Milesi, G., Cesana, B. M., Gheorghiade, M., and Dei Cas, L. (2011). N-3 polyunsaturated fatty acids in the prevention of atrial fibrillation recurrences after electrical cardioversion: a prospective, randomized study. Circulation 124, 1100-1106.

Owen, A. J., Peter-Przyborowska, B. A., Hoy, A. J., and McLennan, P. L. (2004). Dietary fish oil doseand time-response effects on cardiac phospholipid fatty acid composition. Lipids 39, 955-961.

Ozaydin, M., Erdogan, D., Tayyar, S., Uysal, B. A., Dogan, A., Icli, A., Ozkan, E., Varol, E., Turker, Y., and Arslan, A. (2011). N-3 polyunsaturated fatty acids administration does not reduce the recurrence rates of atrial fibrillation and inflammation after electrical cardioversion: a prospective randomized study. Anadolu Kardiyol. Derg. 11, 305-309.

Patel, D., Shaheen, M., Venkatraman, P., Armaganijan, L., Sanchez, J. E., Horton, R. P., Di Biase, L., Mohanty, P., Canby, R., Bailey, S. M., Burkhardt, J. D., Gallinghouse, G. J., Zagrodzky, J.
D., Kozeluhova, M., and Natale, A. (2009). Omega-3 polyunsaturated fatty acid supplementation reduced atrial fibrillation recurrence after pulmonary vein antrum isolation. Indian Pacing Electrophysiol. J. 9, 292-298.

Ramadeen, A., Laurent, G., dos Santos, C. C., Hu, X., Connelly, K. A., Holub, B. J., Mangat, I., and Dorian, P. (2010). n-3 Polyunsaturated fatty acids alter expression of fibrotic and hypertrophic genes in a dog model of atrial cardiomyopathy. Heart Rhythm 7, 520-528.

Sakabe, M., Shiroshita-Takeshita, A., Maguy, A., Dumesnil, C., Nigam, A., Leung, T. K., and Nattel, S. (2007). Omega-3 polyunsaturated fatty acids prevent atrial fibrillation associated with heart failure but not atrial tachycardia remodeling. Circulation 116, 2101-2109.

Saravanan, P., Bridgewater, B., West, A. L., O'Neill, S. C., Calder, P. C., and Davidson, N. C. (2010a). Omega3 fatty acid supplementation does not reduce risk of atrial fibrillation after coronary artery bypass surgery: a randomized, doubleblind, placebo-controlled clinical trial. Circ. Arrhythm. Electrophysiol. 3, 46-53.

Saravanan, P., Davidson, N. C., Schmidt, E. B., and Calder, P. C. (2010b). Cardiovascular effects of marine omega-3 fatty acids. Lancet 376, 540-550.

Sarrazin, J. F., Comeau, G., Daleau, P., Kingma, J., Plante, I., Fournier, D. and Molin, F. (2007). Reduced incidence of vagally induced atrial fibrillation and expression levels of connexins by $\mathrm{n}-3$ polyunsaturated fatty acids in dogs. Am. J. Coll. Cardiol. 50, 1505-1512.

Shen, J., and Johnson, V. M., Sullivan, L. M., Jacques, P. F., Magnani, J. W., Lubitz, S. A., Pandey, S., Levy, D., Vasan, R. S., Quatromoni, P. A., Junyent, M., Ordovas,
J. M., and Benjamin, E. J. (2011). Dietary factors and incident atrial fibrillation: the framingham heart study. Am. Clin. J. Nutr. 93, 261-266.

Skuladottir, G. V., Heidarsdottir, R., Arnar, D. O., Torfason, B., Edvardsson, V., Gottskalksson, G., Palsson, R., and Indridason, O. S. (2011). Plasma n-3 and n-6 fatty acids and the incidence of atrial fibrillation following coronary artery bypass graft surgery. Eur. Clin. J. Invest. 41, 995-1003.

Sorice, M., Tritto, F. P., Sordelli, C., Gregorio, R., and Piazza, L. (2011). N-3 polyunsaturated fatty acids reduces post-operative atrial fibrillation incidence in patients undergoing "on-pump" coronary artery bypass graft surgery. Monaldi Arch. Chest Dis. 76, 93-98.

Suenari, K., Chen, Y. C., Kao, Y. H. Cheng, C. C., Lin, Y. K., Kihara, Y., Chen, Y. J., and Chen, S. A. (2011). Eicosapentaenoic acid reduces the pulmonary vein arrhythmias through nitric oxide. Life Sci. 89, 129-136.

Virtanen, J. K., Mursu, J., Voutilainen, S., and Tuomainen, T. P. (2009) Serum long-chain n-3 polyunsaturated fatty acids and risk of hospital diagnosis of atrial fibrillation in men. Circulation 120, 2315-2321.

Xiao, Y. F., Gomez, A. M., Morgan, J. P., Lederer, W. J., and Leaf, A. (1997). Suppression of voltage-gated L-type Ca2+ currents by polyunsaturated fatty acids in adult and neonatal rat ventricular myocytes. Proc. Natl. Acad. Sci. U.S.A. 94, 4182-4187.

Xiao, Y. F., Sigg, D. C., and Leaf, A. (2005). The antiarrhythmic effect of $n-3$ polyunsaturated fatty acids: modulation of cardiac ion channels as a potential mechanism. Membr. J. Biol. 206, 141-154.

Xiao, Y. F., Wright, S. N., Wang, G. K., Morgan, J. P., and Leaf, A.
(1998). Fatty acids suppress voltagegated na+ currents in HEK293t cells transfected with the alpha-subunit of the human cardiac na+ channel. Proc. Natl. Acad. Sci. U.S.A. 95, 2680-2685.

Xiao, Y. F., Wright, S. N., Wang, G. K., Morgan, J. P., and Leaf, A. (2000). Coexpression with beta(1)-subunit modifies the kinetics and fatty acid block of hH1(alpha) na (+) channels. Am. J. Physiol. Heart Circ. Physiol. 279, H35-H46.

Zhang, Z., Zhang, C., Wang, H., Zhao, J., Liu, L., Lee, J., He, Y., and Zheng, Q. (2011). N-3 polyunsaturated fatty acids prevents atrial fibrillation by inhibiting inflammation in a canine sterile pericarditis model. Int. J. Cardiol. 153, 14-20.

Conflict of Interest Statement: The authors declare that the research was conducted in the absence of any commercial or financial relationships that could be construed as a potential conflict of interest.

Received: 29 February 2012; paper pending published: 19 March 2012; accepted: 02 May 2012; published online: 25 May 2012.

Citation: Rix TA, Mortensen LM and Schmidt EB (2012) Fish, marine n-3 fatty acids, and atrial fibrillation - experimental data and clinical effects. Front. Physio. 3:152. doi: 10.3389/fphys.2012.00152

This article was submitted to Frontiers in Cardiac Electrophysiology, a specialty of Frontiers in Physiology.

Copyright (c) 2012 Rix, Mortensen and Schmidt. This is an open-access article distributed under the terms of the Creative Commons Attribution Non Commercial License, which permits noncommercial use, distribution, and reproduction in other forums, provided the original authors and source are credited. 